\title{
A Comparison of Acoustic Field Measurement by a Microphone and by an Optical Interferometric Probe
}

\author{
R. Bálek, Z. Šlegrová
}

The objective of this work is to show that our optical method for measuring acoustic pressure is in some way superior to measurement using a microphone. Measurement of the integral acoustic pressure in the air by a laser interferometric probe is compared with measurement using a microphone. We determined the particular harmonic components in the acoustic field in the case of relatively high acoustic power in the ultrasonic frequency range.

Keywords: acousto-optics, ultrasonic light diffraction, heterodyne laser interferometer.

\section{Introduction}

Conventional methods for measuring acoustic pressure using a microphone are not quite suitable for some applications. These conventional methods have three drawbacks: they are restricted to about a $100 \mathrm{kHz}$ frequency band, the body of the microphone influences the acoustic field, and contact measurement may sometimes not be possible. These problems are found, e.g., in acoustic measurement in small diameter pipes.

The acoustic field was generated by a power ultrasonic generator with a steel buffer at a frequency of $20 \mathrm{kHz}$ with second and third harmonics of the base signal. Other harmonic components in the acoustic field arose as the result of the relatively high energy in the propagating waves. We solved this problem in optically transparent pipes by using an optical interferometric sensor - a laser probe.

First, we optically measured the acoustic pressure from a piston source in open space, before measuring in the glass waveguide. The optically obtained results were compared with results obtained by the microphone and with piston radiator theory. We intended to prove that the data from the two measurements of higher harmonic components of the acoustic field are in good agreement.

\section{Theory}

\subsection{Acousto-optic interaction}

The process of acousto-optic interaction is described in [1]. When the light ray goes through a volume with variable pressure, the refractive index and the velocity of light propagation differ according to the density changes. We can describe this process as phase modulation of light by acoustic waves. This holds in terms of the Raman-Nath diffraction regime, which is restricted by the Klein-Cook parameter $Q$ and the Raman-Nath parameter $v$ [1] within the bounds

$$
Q \ll 1
$$

and

$$
Q \cdot v \ll 1
$$

The $Q$ and $v$ are defined as follows:

$$
\begin{aligned}
& Q=\frac{2 \pi \lambda L}{n_{0} \Lambda^{2}} \\
& \nu=\frac{2 \pi L \delta n}{\lambda},
\end{aligned}
$$

where $\lambda[\mathrm{m}]$ is the laser light wavelength in a vacuum, $\Lambda[\mathrm{m}]$ is the acoustic wavelength in the interaction medium, $n_{0}[-]$ is the refractive index in a medium without ultrasound, $\delta n[-]$ is the amplitude of the refractive index changes and $L[\mathrm{~m}]$ is the interaction length. The integral phase change of the laser (electromagnetic) wave in the distance $z$ from the acoustic source over the interaction length (in the direction of the $x$-axis) is:

$$
v_{z}(t)=k \mu_{0} \int_{0}^{L} p_{z}(x, t) \mathrm{d} x,
$$

where $k\left[\mathrm{~m}^{-1}\right]$ is the laser wave number in a vacuum, $\mu_{0}\left[\mathrm{~Pa}^{-1}\right]$ is the adiabatic piezo-optic coefficient, calculated from the refractive index of the medium and $p_{z}(x, t)[\mathrm{Pa}]$ is the acoustic pressure at a distance $z$ from the acoustic source at any point on the $x$-axis. The system of coordinates is defined in Fig. 1.

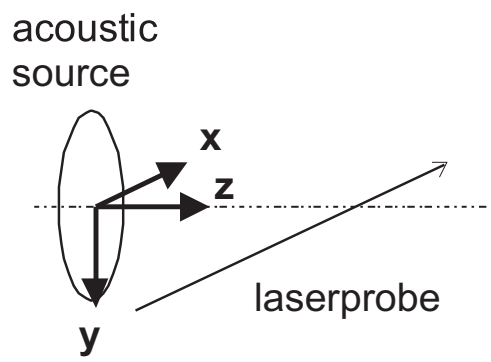

Fig. 1: System of coordinates

Let us assume for simplicity only two higher harmonic components in the acoustic signal that correspond to two plane acoustic waves. The same steps could be followed in the case of a more complex signal. The time dependence of the acoustic pressure can be written as:

$p_{z}(x, t)=p_{0 z 1} \sin \left(\omega_{A 1} t+\phi_{A 1}\right)+p_{0 z 2} \sin \left(\omega_{A 2} t+\phi_{A 2}\right)$. 
The integral phase change (5) is in terms of plane waves equal to:

$v_{z}(t)=k L \mu_{0} \cdot\left[p_{0 z 1} \sin \left(\omega_{A 1} t+\phi_{A 1}\right)+p_{0 z 2} \sin \left(\omega_{A 2} t+\phi_{A 2}\right)\right]$,

or, more simply,

$$
\begin{aligned}
& v_{z}(t)=v_{z 1}(t)+v_{z 2}(t) \\
& v_{z}(t)=k \mu_{0} p_{i z}(t),
\end{aligned}
$$

where $p_{i z}(t)$ is the integral value of the acoustic pressure.

The interaction of a laser beam with the acoustic wave causes spatial diffraction, which can be expressed by means of diffraction angles. Diffraction angles $\Theta_{n}$ of the particular orders of diffraction are:

$$
\Theta_{n}=n \frac{\lambda}{\Lambda n_{0}} .
$$

where $n$ is the diffraction index of the interaction media.

For this low frequency acoustic field there are negligible angles of diffraction, so we can consider all $n$ orders of diffraction as a single laser beam:

$$
\begin{gathered}
S=S_{0} \sum_{m=-\infty}^{+\infty} \sum_{n=-\infty}^{+\infty} J_{m}\left(v_{01 z}\right) J_{n}\left(v_{02 z}\right) \exp \left\{-\mathrm{j}\left[\left(\omega_{L}-m \omega_{A 1}+\right.\right.\right. \\
\left.\left.\left.-n \omega_{A 2}\right) t+\phi_{S}+m \phi_{A 1}+n \phi_{A 2}\right]\right\}
\end{gathered}
$$

where $S_{0}$ is the amplitude of the incoming electromagnetic wave, $J_{m}, J_{n}$ are Bessel functions of the first kind, of the order of $m, n$ and $v_{01 z}, v_{02 z}$ are Raman-Nath parameters corresponding to the values of the amplitudes of light phase changes $v_{1 z}(t), v_{2 z}(t)$, due to acoustic waves.

\subsection{Laser interferometric detection}

We chose the heterodyne laser interferometry method for detection of acoustic pressure. The advantages of this method are the high signal to noise ratio $(80 \mathrm{~dB})$ and the possibility of absolute determination of the signal level [2]. The output signal of the interferometer carries information about the phase changes of the detected laser beam. This information is transformed from the optical band to a much more acceptable lower frequency range. The detailed characteristics of the output signal created by the simple harmonic acoustic wave are introduced in [3]; this paper also describes methods for determining the amplitude of the pressure from the frequency spectrum of this signal. More complex acoustic fields produce more complex interferometer output signals. We cannot compute the amplitude of the pressure from the simple ratio of individual signal amplitudes corresponding to certain frequencies. It is necessary to use the whole signal frequency spectrum. We solved this problem in a way analogous to the processing of frequency modulated radio signals. After the phase demodulation the obtained amplitudes are directly proportional to the averaged values of the acoustic pressure of the individual harmonic components.

\section{Experiments}

\subsection{Description of the setup}

\subsubsection{Ultrasonic generator}

Ultrasonic waves are generated at base frequency $20.5 \mathrm{kHz}$ by a couple of piezo-electric transducers connected at one end with a steel buffer, which radiates as a piston generator into the ambient air. The acoustic signal is a nearly sinusoidal wave with small second and third harmonics of the base signal. Two buffers were used. The first, labeled as N, has a constant circular cross-section. The cross section of the other buffer decreases toward the radiated end. The characteristics of both are shown in Table 1. The near-field length was calculated from the formula for a circular piston radiator [4]. The output power of the generator can be changed from $20 \%$ to $100 \%$.

Table 1: Parameters of the buffers

\begin{tabular}{|l|c|c|c|}
\hline $\begin{array}{l}\text { Type } \\
\text { of } \\
\text { buffer }\end{array}$ & $\begin{array}{c}\text { Radius of } \\
\text { radiated aperture } \\
R[\mathrm{~mm}]\end{array}$ & $\begin{array}{c}\text { Base } \\
\text { frequency } \\
f_{1}[\mathrm{~Hz}]\end{array}$ & $\begin{array}{c}\text { Near field } \\
\text { distance } \\
\ell_{0}[\mathrm{~mm}]\end{array}$ \\
\hline $\mathrm{N}$ & 15 & $20.5 \cdot 10^{3}$ & 9.00 \\
\hline $\mathrm{K}$ & 8 & $20.5 \cdot 10^{3}$ & 0.37 \\
\hline
\end{tabular}

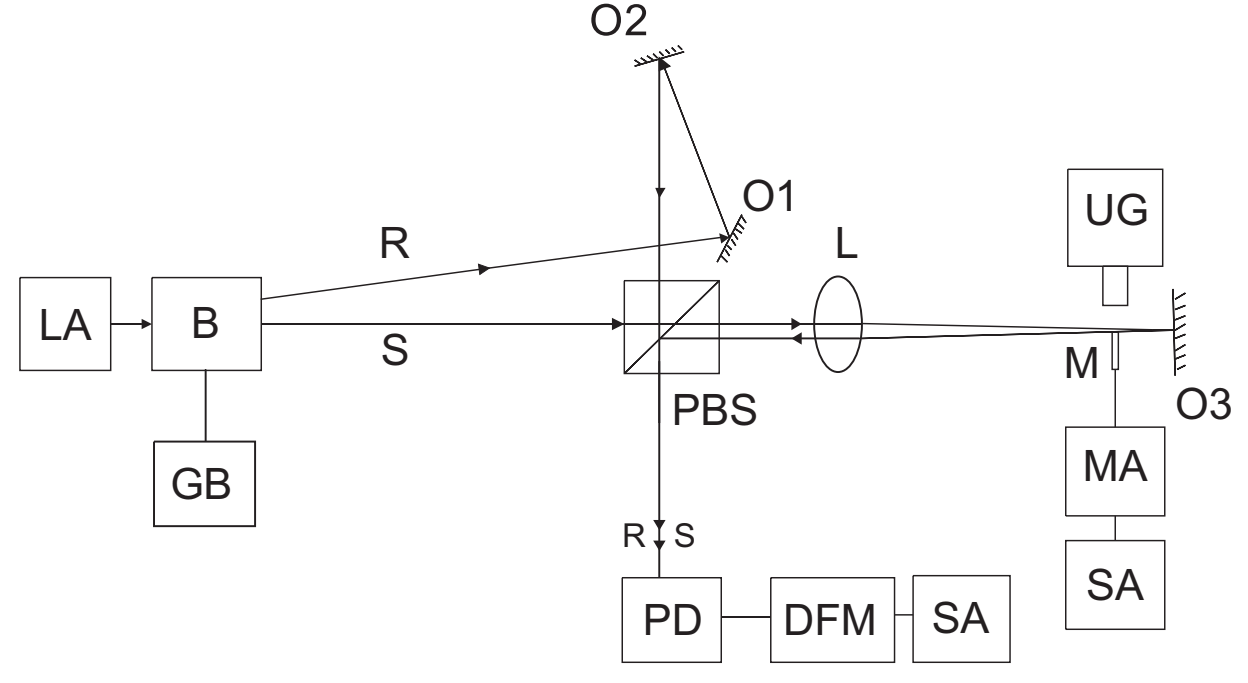

Fig. 2: Schematic diagram:

B- Bragg cell, DFM- frequency demodulator, GB- Bragg cell signal generator, L- lens, LA- He-Ne laser, M- microphone, MA- amplifier, O1,O2,O3- mirrors, PBS- polarizing beam splitter, PD- photodiode, SA- spectral analyzer, UG-ultrasonic generator with piston buffer 


\subsubsection{Measurement by microphone}

The level of the generated signal was measured by a $1 / 8^{\prime \prime}$ G.R.A.S. Type 40DP microphone with a 26AC preamplifier. The bandwidth of this microphone within a $0.5 \mathrm{~dB}$ drop is $100 \mathrm{kHz}$. The acoustic field was scanned at different distances from the aperture of the radiator by means of a computer controlled scanning 3 -axis bridge. The scanning area is $(90 \times 90) \mathrm{mm}$ in the $x-y$ plane with a minimum possible step of $50 \mu \mathrm{m}$. The signal from the microphone was measured with a Hewlett Packard 8560E spectrum analyzer and recorded by a computer. Position data was also recorded.

\subsubsection{Optical setup and signal processing}

A schematic diagram of the optical measurement system is shown in Fig. 2. A detailed description of this apparatus is given in [3]. The Hewlett Packard 8593E spectral analyzer, which has a frequency demodulator (DFM) with variable bandwidth, demodulates the signal from the photodiode PD. The demodulated signal is recorded by the other spectral analyzer SA (Hewlett Packard 8560E).

\subsection{Raman-Nath regime assessment}

The constants from Table 2 were used for computing [5].

Table 2: Constants used for computing

\begin{tabular}{|l|c|c|}
\hline & Amplitudes & Units \\
\hline refraction index of air & 1.000276 & - \\
\hline velocity of ultrasound & 343.6 & $\mathrm{~m} \cdot \mathrm{s}^{-1}$ \\
\hline piezo-optic coefficient & $2 \cdot 10^{-9}$ & $\mathrm{~Pa}^{-1}$ \\
\hline laser wavelength & 632.8 & $\mathrm{~nm}$ \\
\hline
\end{tabular}

As was stated above, proof of the validity of using acousto-optic interaction requires that we satisfy all conditions of the Klein-Cook and Raman-Nath parameters (1), (2). The amplitude of the integral pressure at distance $\ell_{0}$ on the $z$-axis and the amplitudes obtained from piston radiator theory are shown in Table 3. The limit value of the Raman-Nath parameter $v=1$ was used. The Klein-Cook parameter $Q$ is also presented. The interaction length $L$, defined as the width of the acoustic field, perpendicular to the z-axis, had to be doubled (the laser light goes through twice).

Table 3: Raman-Nath regime

\begin{tabular}{|l|c|c|c|}
\hline Buffer & $p_{\text {il0 }}[\mathrm{Pa} \cdot \mathrm{m}]$ & $p_{10 \max }[\mathrm{Pa}]$ & $Q[-]$ \\
\hline $\mathrm{N}$ & 25 & 1475 & $8.43 \cdot 10^{-4}$ \\
\hline $\mathrm{K}$ & 25 & 2268 & $4.5 \cdot 10^{-4}$ \\
\hline
\end{tabular}

\section{Results, discussion}

\subsection{A comparison of measurement by microphone with results from piston radiator theory}

Fig. 3 shows examples of acoustic field distribution, with effective values of acoustic pressure, in the $x-y$ plane measured by a microphone. Fig. 4 compares the measured amplitudes

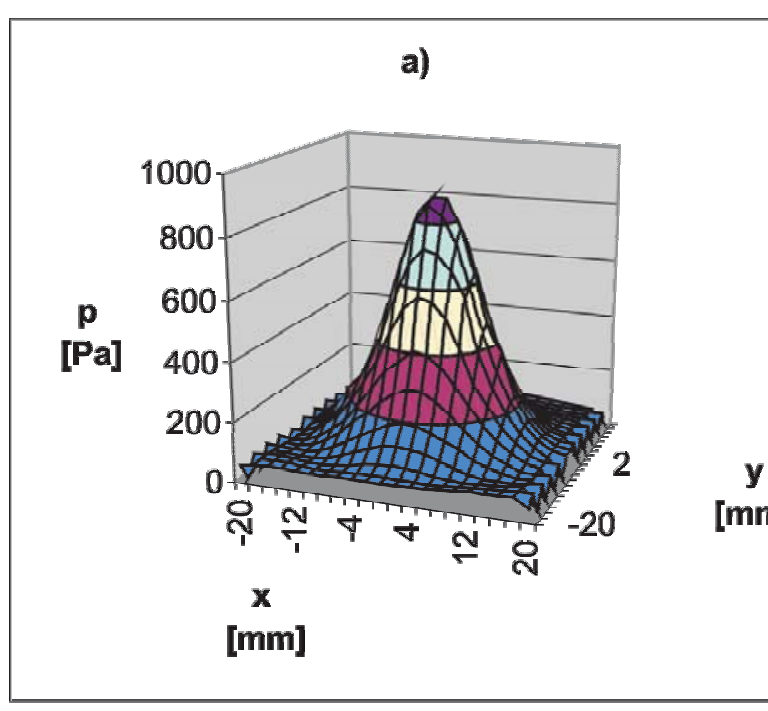

b)

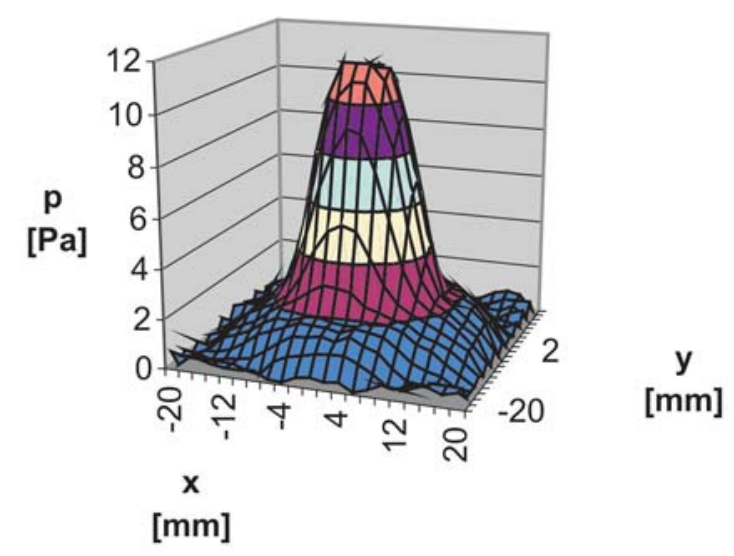

c)

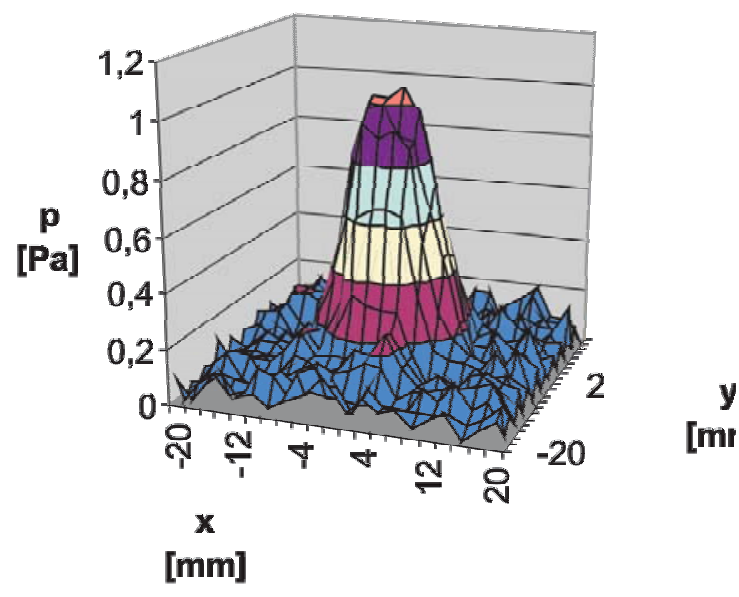

Fig. 3: Microphone measured acoustic field distribution in the $x-y$ plane at distance $z=6.5 \mathrm{~mm}$ from the buffer $\mathrm{K}$ with $60 \%$ power from the generator:

a) on base frequency $\mathrm{f}_{1}=20.5 \mathrm{kHz}$

b) on second harmonic frequency $\mathrm{f}_{2}=41 \mathrm{kHz}$

c) on third harmonic frequency $\mathrm{f}_{3}=61.5 \mathrm{kHz}$ 


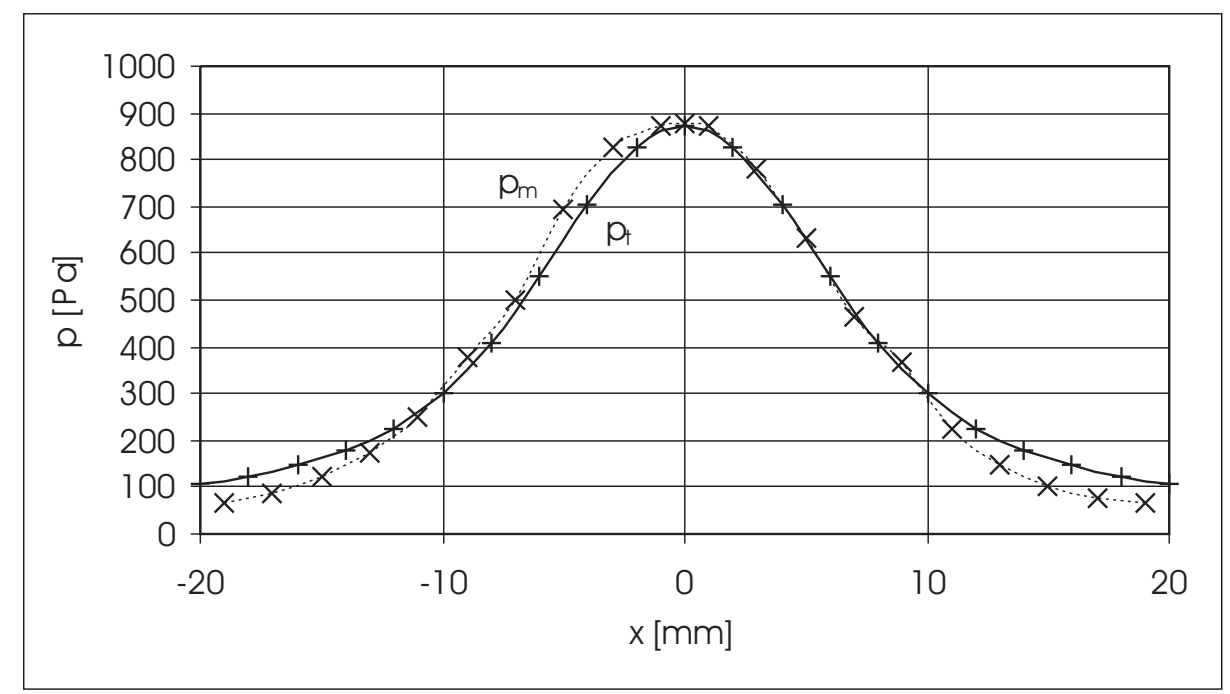

Fig. 4: Dependence of acoustic pressure from K buffer on the x-coordinate at distance $z=6.5 \mathrm{~mm}, f_{1}=20.5 \mathrm{kHz}$, generator power $60 \%$. $p_{t}$ - from theory, $p_{m}$ - from experiment with microphone.

Table 4: Results

\begin{tabular}{|c|c|c|c|c|c|c|c|c|c|}
\hline \multirow[t]{2}{*}{ buffer } & \multirow{2}{*}{$\begin{array}{c}z \\
{[\mathrm{~mm}]}\end{array}$} & \multirow{2}{*}{$\begin{array}{c}\text { power } \\
{[\%]}\end{array}$} & \multirow{2}{*}{$\stackrel{f}{f}$} & \multicolumn{3}{|c|}{$p_{\mathrm{i}}[\mathrm{Pa} \cdot \mathrm{m}]$ over center of buffer, $y=0$} & \multicolumn{3}{|c|}{$p_{\mathrm{i}}[\mathrm{Pa} \cdot \mathrm{m}]$ over edge of buffer, $y=R$} \\
\hline & & & & laser & microphone & theory & laser & microphone & theory \\
\hline \multirow[t]{2}{*}{$\mathrm{N}$} & \multirow[t]{2}{*}{9.0} & \multirow[t]{2}{*}{20} & 20.5 & 1.575 & 1.77 & 1.71 & 0.59 & 0.60 & 0.68 \\
\hline & & & 41.0 & & 0.0044 & 0.043 & & 0.0017 & 0.0017 \\
\hline \multirow[t]{2}{*}{$\mathrm{K}$} & \multirow[t]{2}{*}{6.5} & \multirow[t]{2}{*}{60} & 20.5 & 15.22 & 15.55 & 15.83 & 10.70 & 8.00 & 9.20 \\
\hline & & & 41.0 & 0.13 & 0.21 & 0.14 & 0.09 & 0.10 & 0.063 \\
\hline
\end{tabular}

on the $x$-axis with piston radiator theory. The theory is derived in [4] and the individual amplitudes are related to the maximum measured value on the $z$-axis. The theory is derived for an ideal piston radiator placed in the infinity plane. A real buffer radiates to free space. This explains the difference between the two curves with increasing $x$.

\subsection{Optical method measurement, comparison of results}

We performed many measurements to check the laser probe measurements with those using the microphone. Microphone measurements had to be performed over the entire
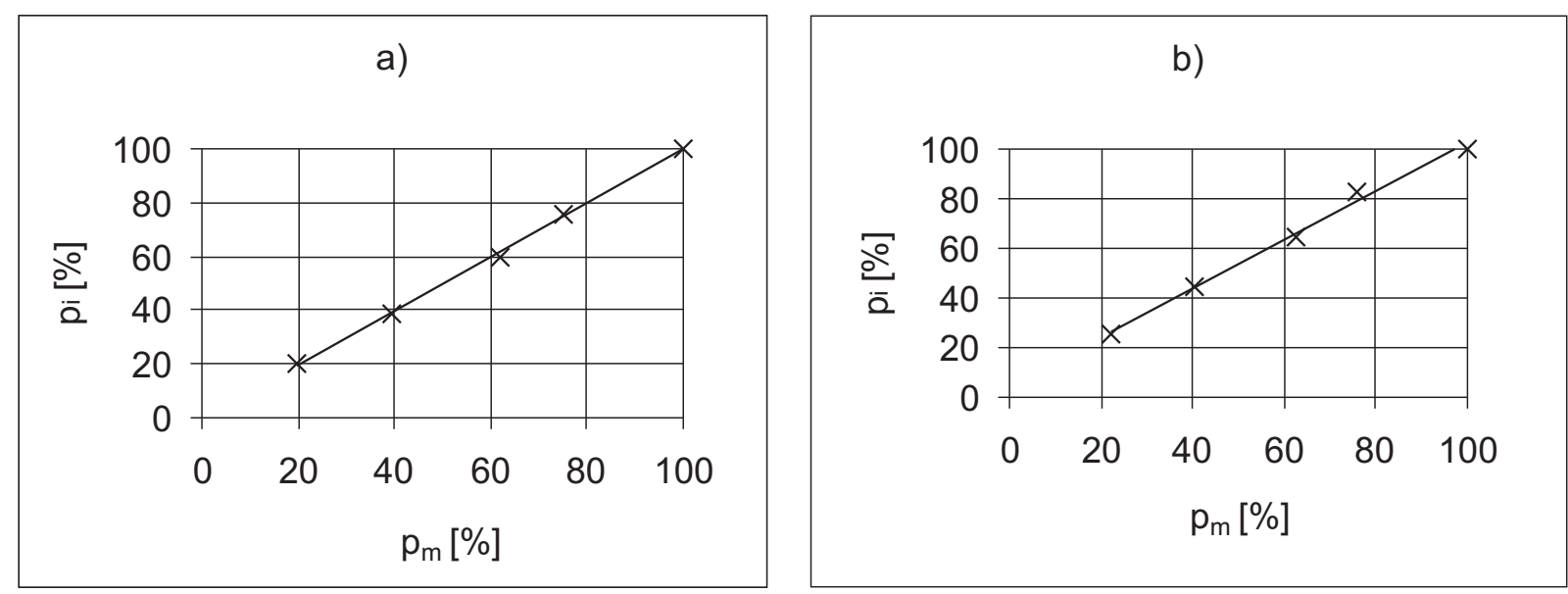

Fig. 5: Dependence of optically measured integral pressure in the direction perpendicular to the $z$-axis of buffer on the measured pressure of the microphone on the $z$-axis at frequency $f=20.5 \mathrm{kHz}$ : a) buffer K, $z=6.5 \mathrm{~mm}$, b) buffer $\mathrm{N}, z=9 \mathrm{~mm}$. 
length of the acoustic field to obtain integral values that were similar to those obtained by the optic method. The transfer constant of frequency modulation was estimated from a calculation of the simple harmonic signal demodulation. Optical measurement was performed in the presence and in the absence of the microphone to determine whether the microphone influences the acoustic field.

Examples of the integral pressure obtained from the measurements and from piston radiator theory are introduced in Table 4.

Table 4 shows that the integral values obtained by all three methods are much closer for the central acoustic beam area and for higher acoustic pressures. The central area is better determined because of the maximum of acoustic pressure. At the edge, small coordinate changes produced large changes in the obtained amplitudes. In addition, the measured data are influenced by noise for small signals. The amplitude of the integral pressure for the second harmonic component was below the pitch of sensitivity for buffer $\mathrm{N}$, and is therefore missing. The minimum detected amplitude of integral pressure was $0.01 \mathrm{~Pa} \cdot \mathrm{m}$. Another deviation can be explained by the finite size of the body of the microphone. The measurements could not be made at the same place and time because the microphone would stop the laser beam. Also, the long term stability of the power generator and the settings of the output level could cause some errors. In spite of all these factors, the results are generally in agreement.

The $z$-axis dependence of integral pressure $p_{i}$ obtained from the laser probe on the pressure measured by microphone $p_{m}$ is displayed in Fig. 5. The first graph is for buffer $\mathrm{K}$ and the second's for $\mathrm{N}$ at the basic frequency of radiation. The acoustic pressure is related to $100 \%$ of the power generator setting. The acoustic pressure measured by the laser probe is linearly dependent on the pressure measured by the microphone along the $z$-axis of the acoustic field.

\section{Conclusion}

Our results prove that it is possible to use a laser probe for measuring integral acoustic pressure. Accuracy in placement of the probes (mainly the microphone) and measurements at different times produced deviations in the results obtained using the different systems.

To determine the acoustic pressure at a specific point the tomography method can be used.

The results obtained in this study will help us to measure acoustic fields in glass pipes where a microphone cannot be used.

\section{Acknowledgements}

This research has been supported by Research Project MSM 212300016 and grant CTU0206313.

\section{Reference}

[1] Korpel, A.: Acousto-Optics. New York and Basel: Marcel Dekker, INC., 1988, p. 43-93.

[2] Bálek, R., Šlegrová, Z.: Modeling and Measurement Laser Induced Surface Displacement. Euromech 419 Colloquium, Institute of Thermomechanics AS CR, 2000, p. 32.

[3] Bálek, R., Šlegrová, Z.: Interferometric Measurement of Acoustic Pressure. ATP 2001, VUT Brno, 2001, p. 41-45.

[4] Škvor, Z.: Akustika a elektroakustika. Praha: Academia, 2001, p. 120-129.

[5] American Institute of Physics Handbook. New York: McGraw-Hill, 1972.

Rudolf Bálek, Ph.D., Assoc. Prof.

phone: +420224352384

e-mail: balek@feld.cvut.cz

Zuzana Šlegrová, M.Sc.

phone: +420224352386

e-mail:slegroz@feld.cvut.cz

Department of Physics

Czech Technical University in Prague

Faculty of Electrical Engineering

Technická 2

16627 Praha 6, Czech Republic 